\title{
Primary Ovarian Lymphoma: A Case Report and Review of Literature
}

\author{
Kumar Narendra • Kumar Ritesh • Bera Anjan • \\ Srinivasan Radhika $\cdot$ Sharma Suresh Chander
}

Received: 4 August 2011/Accepted: 23 April 2012/Published online: 5 October 2012

(C) Federation of Obstetric \& Gynecological Societies of India 2012

\section{Introduction}

Involvement of the ovary by malignant lymphoma is well known as a late manifestation of disseminated disease. However, primary ovarian lymphoma (POL) is rare, accounting for $0.5 \%$ of all non-Hodgkin's lymphoma and $1.5 \%$ of all ovarian neoplasms [1]. We present a case of ovarian non-Hodgkin's lymphoma, manifesting like an advanced ovarian cancer, which was managed by surgery and chemotherapy (Fig. 1).

\section{Case Report}

A 45-year-old premenopausal woman (gravida 1, para 1) presented with history of pain in right side of lower abdomen, dull aching in character for 4 months which increased in severity progressively. It was associated with feeling of heaviness in lower abdomen for 2 months. She

Kumar N. · Kumar R. ( $₫)$, Senior Resident .

Bera A. S Sharma S. C.

Department of Radiotherapy and Oncology, Regional Cancer Centre, Postgraduate Institute of Medical Education and Research, Chandigarh, India

e-mail: riteshkr9@gmail.com

Srinivasan R.

Department of Pathology, Postgraduate Institute of Medical

Education and Research, Chandigarh, India had no other systemic complains. She had normal and regular menstrual cycles with one live issue 17 years before. No other significant co-morbidities were present (Fig. 2). On examination, she had a large abdominopelvic mass present which was firm to hard in consistency and arose from the adnexal region. CECT abdomen and pelvis showed a heterogeneous mass in lower abdomen with minimal ascites. CA-125 level was 85.93 (Fig. 3). Chest $\mathrm{X}$-ray and thyroid profile was normal. She underwent exploratory laparotomy with total abdominal hysterectomy and bilateral salpingo-oophorectomy. Intraoperatively, there was a $20 \times 15 \mathrm{~cm}$ size irregular mass, firm in consistency which was seen arising from right ovary. Final histopathology reported it as non-Hodgkin lymphoma (NHL) of right ovary. Morphologically, it was classified as diffuse large cell B-cell lymphoma (DLBCL). On immunohistochemistry, CD 20 and LCA were positive, while CK, PLAP, and CD 3 were negative. With the histopathological diagnosis of right ovarian NHL, the patient underwent further staging evaluation (Fig. 4)). No systemic lymphadenopathy was found. CECT neck, chest, abdomen, and pelvis were normal except for post-hysterectomy status. Bone marrow aspiration and biopsy did not showed infiltration. CA-125 was 9.4 postoperatively. Patient was staged as Ann Arbor Stage IE and was started on systemic chemotherapy with R-CHOP regimen comprising rituximab, cyclophosphamide, vincristine, and prednisolone in standard doses. Patient has received 6th cycle in February 2011 and is on regular follow-up with no clinical and radiological evidences of disease. 


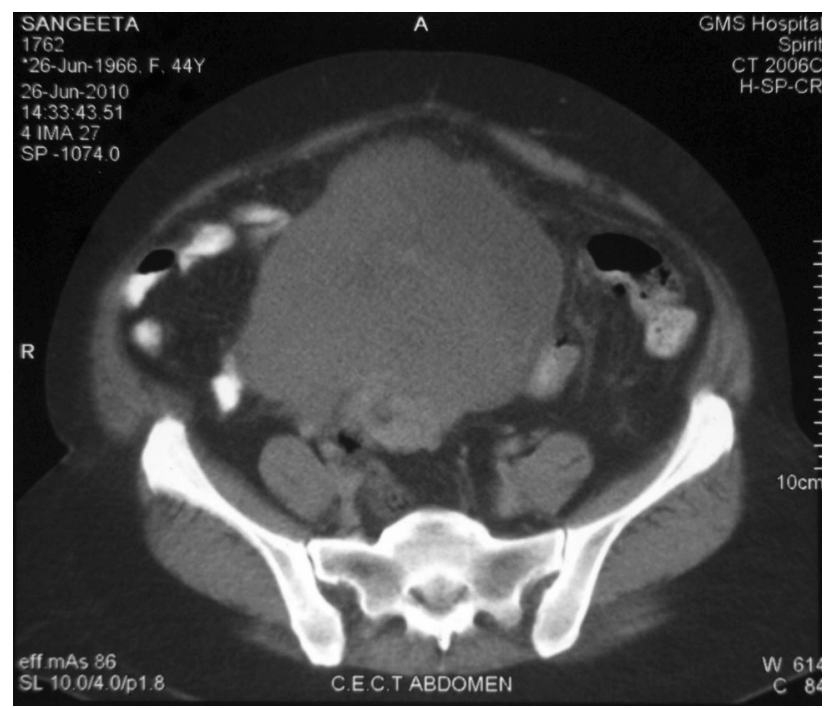

Fig. 1 CECT scan showing ovarian mass-axial section

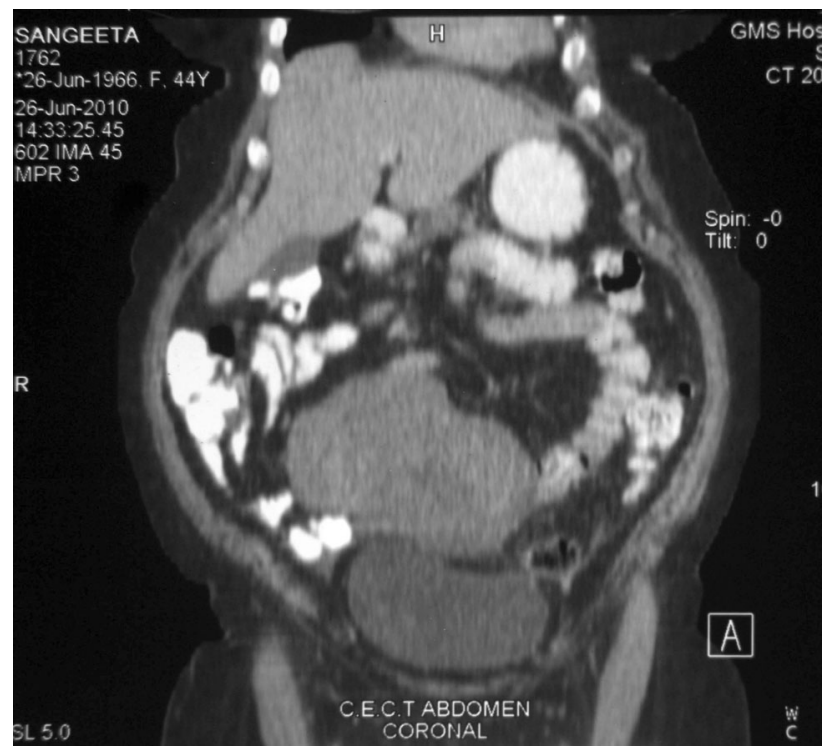

Fig. 2 CECT scan showing ovarian mass-coronal section

\section{Discussion}

Malignant lymphoma is revealed by an ovarian mass in $<1 \%$ of all NHL, while autopsies performed on patients with NHL show an ovarian enlargement in 7-26\% [2]. POL is still much rarer and represents $0.5 \%$ of NHL and $1.5 \%$ of all ovarian neoplasms [1]. Diffuse lymphoma and a B-cell phenotype are the most common histological type and phenotype, respectively $[1,3,4]$.

The distinction between primary and secondary lymphomas is usually made postoperatively, when the diagnosis of ovarian lymphoma is established and when its

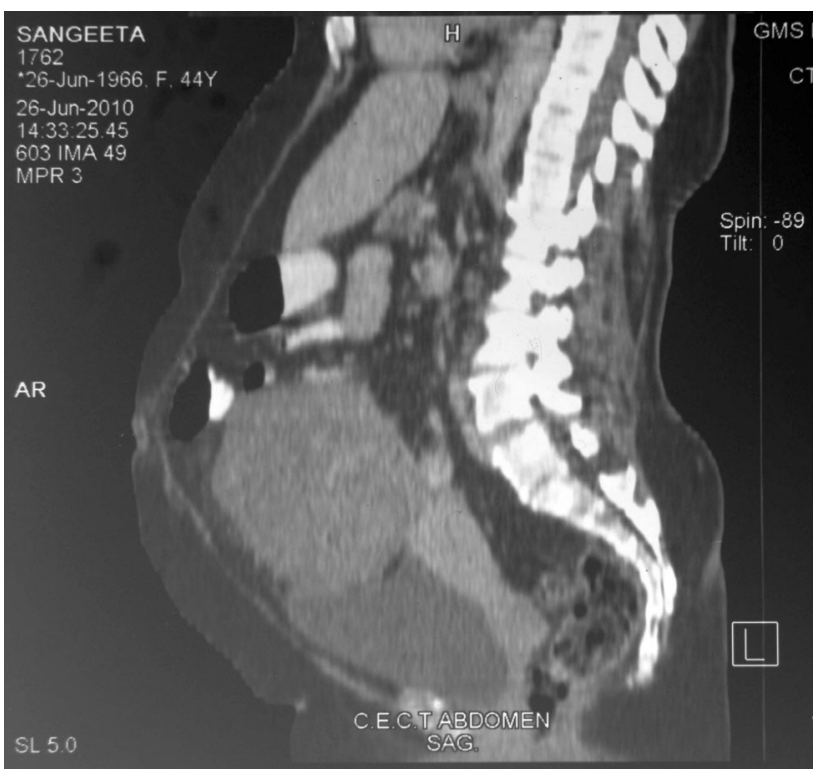

Fig. 3 CECT scan showing ovarian mass-sagittal section

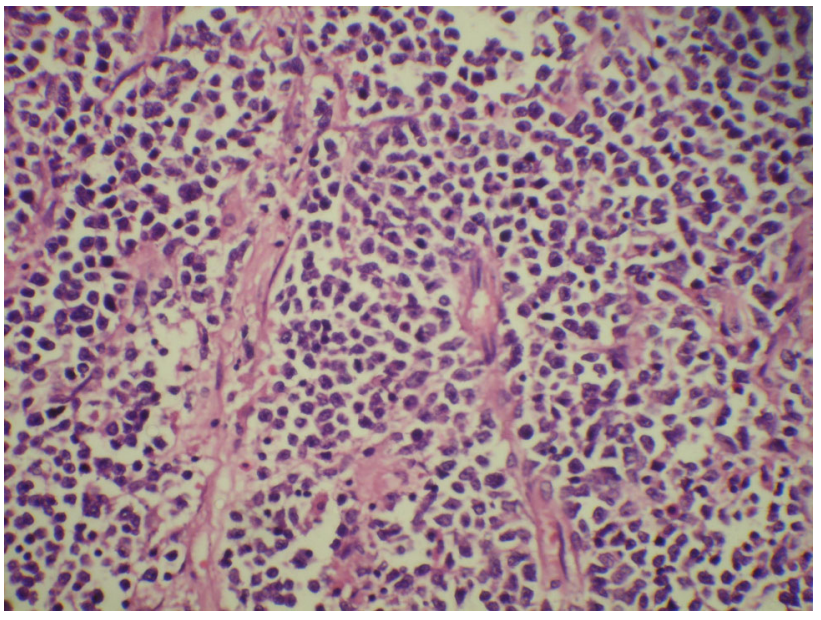

Fig. 4 Microphotograph showing diffuse sheets of large atypical lymphoid cells (hematoxylin and eosin stain)

extension is evaluated. Fox et al. [5] have proposed the stringent criteria for the diagnosis of a POL. Primary and secondary ovarian lymphomas differ in terms of prognosis. Contrary to POL, patients with an occult nodal lymphoma presenting as an ovarian mass have a poor outcome with 5 -year survival rate ranging from 7 to $38 \%[4,6,7]$.

Most patients of POL are young, with a median age of 42-47 years [1]. Fever, emaciation, or night sweats have to be investigated during the patient's interview. Clinical examination may reveal a palpable adenopathy, or a liver or spleen infiltration [1,7]. None of these clinical signs, except a relatively young age, was found in our patient.

Ferrozzi et al. [8] reported eight patients with ovarian NHL and assessed their most typical imaging patterns. 


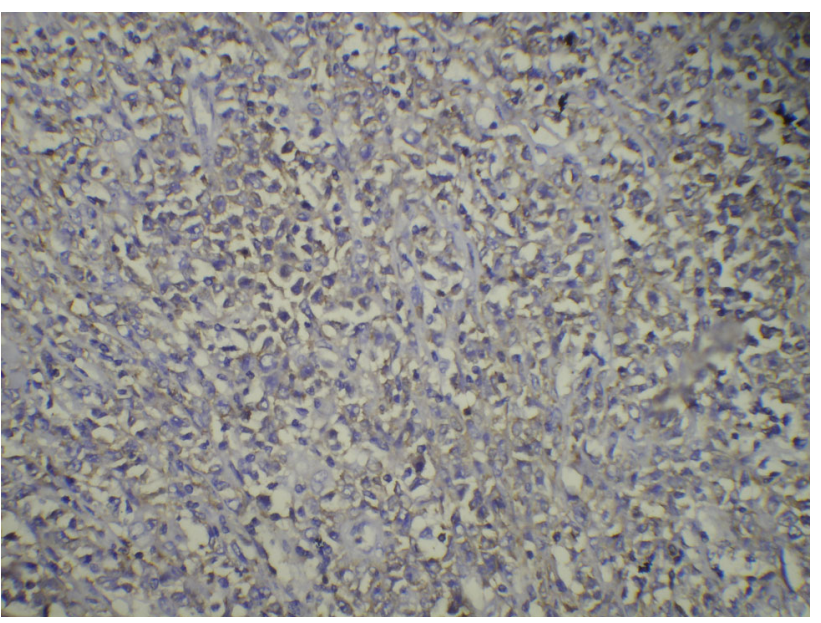

Fig. 5 Immunohistochemistry for LCA

Ovarian lymphomas were frequently bilateral and homogeneous, without ascites, and the tumors always exceeded $5 \mathrm{~cm}$ in diameter. Ultrasonography showed homogeneous, hypoechoic, and mildly vascularized tumors. In all cases, computed tomography (CT) revealed clear-cut hypodense lesions, with mild contrast enhancement. In our patient, imaging was rather suggestive of an ovarian carcinoma. CT scan showed a heterogeneous mass, reshaped by several necrotic centers (Fig. 5).

Lymphomas are treated with chemotherapy, using a regimen based on the cytological type of the NHL. There is not a standard treatment protocol for primary ovarian NHL because of its rarity. The best option seems to be chemotherapy according to the specific histology. A CHOP regimen (cyclophosphamide, adriamycin, vincristin, and prednisone) is usually administered.

Yamada et al. report a case with a diffuse large B-cell ovarian malignant lymphoma, which presented as an advanced carcinoma successfully treated with eight IV CHOP regimens. A mixture of $100 \mathrm{mg}$ of cisplatin, $400 \mathrm{mg}$ of etoposide, and $1,000 \mathrm{ml}$ of saline solution was also administered in the peritoneal cavity because an undifferentiated carcinoma was suspected at the histological examination of the frozen section. The patient was alive and disease-free 6 years after the chemotherapy [6].

Niitsu et al. [4] obtained in a 54-year-old woman the complete remission of a follicular lymphoma (grade 1, stage IIE) with a combination of cyclophosphamide, vincristin, bleomycin, etoposide, doxorubicin, and prednisolone (cycl OBEAP) (Fig. 6).

Dimopoulos et al. [1] reported complete remissions and long-term survivals in patients treated by combination chemotherapy appropriate to each histologic type. In all their patients, the diagnosis could only be established after a laparotomy, whereas the clinical findings suggested an epithelial ovarian neoplasm. Chemotherapy regimens

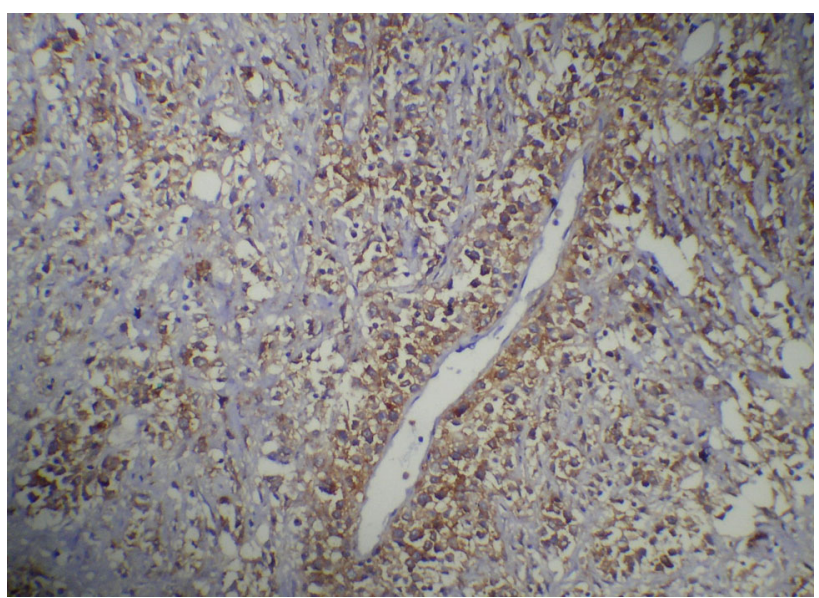

Fig. 6 Immunohistochemisty for CD 20 showing strong positivity in tumor cells

included CHOP regimen or variants of CHOP. The authors obtained a complete remission in $64 \%$ of their patients. Long-term disease-free survival and overall survival were noted in 46 and $57 \%$, respectively.

Our patient received chemotherapy with R-CHOP regimen for six courses and is clinically and radiologically disease-free.

POL is rare and mimics other common gynecologic tumors. Outcome seems to be determined by its histologic type and phenotype, and by the chemotherapy regimen. Stage and mode of presentation also influence its prognosis.

\section{References}

1. Dimopoulos M. Primary ovarian non-Hodgkin's lymphoma: outcome after treatment with combination chemotherapy. Gynecol Oncol. 1997;64(3):446-50.

2. Rosenberg SA, Diamond HD, Jaslowitz B, et al. Lymphosarcoma: a review of 1269 cases. Medicine (Baltimore). 1961;40:31-84.

3. Vang R, Medeiros LJ, Warnke RA, et al. Ovarian non-Hodgkin's lymphoma: a clinicopathologic study of eight primary cases. Mod Pathol. 2001;14(11):1093-9.

4. Niitsu N, Nakamine H, Hayama M, et al. Ovarian follicular lymphoma: a case report and review of the literature. Ann Hematol. 2002;81(11):654-8.

5. Fox H, Langley FA, Govan AD, et al. Malignant lymphoma presenting as an ovarian tumour: a clinicopathological analysis of 34 cases. Br J Obstet Gynaecol. 1988;95(4):386-90.

6. Yamada T. A case of malignant lymphoma of the ovary manifesting like an advanced ovarian cancer. Gynecol Oncol. 2003;90(1):215-9.

7. Türken A, Ciftci AO, Akçören Z, et al. Primary ovarian lymphoma in an infant: report of a case. Surg Today. 2000;30(3):305-7.

8. Ferrozzi F, Catanese C, Uccelli M, et al. Ovarian lymphoma. Findings with ultrasonography, computerized tomography and magnetic resonance. Radiol Med. 1998;95(5):493-7. 